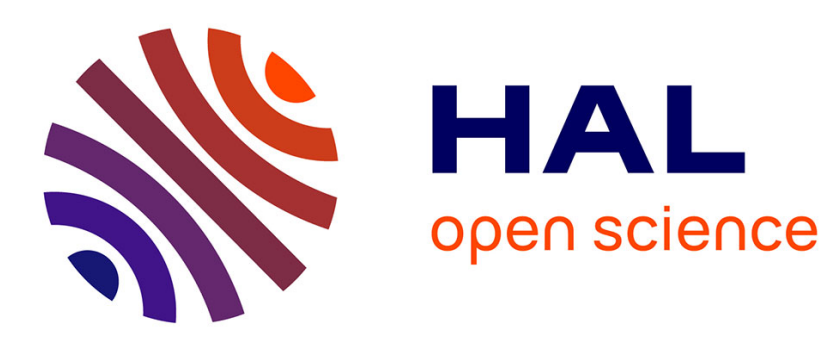

\title{
La transformation logarithme des processus stochastiques, ergodicité
}

\author{
M. Maurin
}

\section{To cite this version:}

M. Maurin. La transformation logarithme des processus stochastiques, ergodicité. Journal de Physique IV Proceedings, 1994, 04 (C5), pp.C5-1353-C5-1356. 10.1051/jp4:19945301 • jpa-00253015

\section{HAL Id: jpa-00253015 https://hal.science/jpa-00253015}

Submitted on 1 Jan 1994

HAL is a multi-disciplinary open access archive for the deposit and dissemination of scientific research documents, whether they are published or not. The documents may come from teaching and research institutions in France or abroad, or from public or private research centers.
L'archive ouverte pluridisciplinaire HAL, est destinée au dépôt et à la diffusion de documents scientifiques de niveau recherche, publiés ou non, émanant des établissements d'enseignement et de recherche français ou étrangers, des laboratoires publics ou privés. 


\title{
La transformation logarithme des processus stochastiques, ergodicité
}

\author{
M. MAURIN
}

INRETS-LEN, Case 24, 69675 Bron cedex, France

\begin{abstract}
Classical definition of equivalent noise levels $\mathrm{Leq}_{\Delta \mathrm{t}}$ (see below eq.1) implies some special time Processes with Stationary Increments, Independent (PSII), or no (PSI). With PSII $\mathrm{Leq}_{\Delta t}$ are independent then tritely ergodic, but for mere PSI Leq $\mathrm{St}_{\mathrm{t}}$ ar not independent nor ergodic. As Leq are transformed random variables with logarithm, logarithm transform deserves to be investigated in relation to stochastic processes properties, and specially to ergodicity.

In this way it is shown the logarithm of a positive stationary ergodic process $Z_{t}$ is itself stationary and ergodic under very weak conditions.
\end{abstract}

\section{INTRODUCTION.}

La définition des indices de bruit équivalents $\operatorname{Leq}_{\Delta t}=10 \log \left\{\frac{1}{\Delta t} \int_{t}^{t+\Delta t}\left(p_{u} / p_{0}\right)^{2} d u\right\}$ (eq.1) en acoustique de l'environnement suggère l'emploi de processus spéciaux pour la (sur-)pression acoustique $p_{t}$, sa puissance $\mathrm{p}_{\mathrm{t}}{ }^{2}$ et son énergie $\mathrm{X}_{\mathrm{t}}=\int \mathrm{p}_{\mathrm{u}}{ }^{2} \mathrm{du},\left(\mathrm{p}_{0}=210^{-5} \mathrm{~Pa}\right)$. Par exemple les Processus à Accroissements Indépendants et Stationnaires de Lévy (PAIS) [1] positifs et du 2ème ordre pour $\mathrm{X}_{\mathfrak{t}}$, les accroissements $\Delta X_{i}={ }_{t+i \Delta t}^{t+(i+1) \Delta t} p_{u}{ }^{2} d u$ auxquels nous sommes conduits sont en outre trivialement ergodiques, de même que leur logarithme [2]. Les PAS sont plus nombreux, ils sont également intéressants et leurs accroissements $\Delta \mathrm{X}_{\mathrm{i}}$ sont encore ergodiques sous de larges conditions [3]. Se pose ainsi la question de l'ergodicité des accroissements transformés $\operatorname{Leq}_{\Delta t, i}=10 \log \frac{\Delta X_{i}}{p_{0}^{2} \Delta t}$ par le logarithme, laquelle est utile pour le traitement statistique des $\operatorname{Leq}_{\Delta t, i}$ successifs sur tous intervalles $[\mathrm{t}+\mathrm{i} \Delta \mathrm{t}, \mathrm{t}+(\mathrm{i}+1) \Delta \mathrm{t}]$.

Le contexte acoustique étant rappelé il est plus simple de prendre de nouvelles notations ; en substance il s'agit d'étudier une suite d'accroissements transformés $\mathrm{L}_{\mathrm{i}}=\varphi\left(\Delta_{\mathrm{i}}\right)$ avec $\Delta_{\mathrm{i}}$ l'accroissement d'un processus $\mathrm{Z}_{\mathrm{t}}$ sur $[\mathrm{t}+\mathrm{i} \Delta \mathrm{t}, \mathrm{t}+(\mathrm{i}+1) \Delta \mathrm{t}]$ dans le cas particulier de l'application logarithme.

Pour cela nous avons besoin en premier d'un résultat technique sur l'ergodicité du signal transformé $\mathrm{L}_{\mathfrak{t}}=$ $\varphi\left(Z_{\mathfrak{t}}\right)$ à partir d'un signal $Z_{\mathfrak{t}}$ stationnaire du second ordre, positif et ergodique. Nous montrons ci-après que moyennant des conditions très larges le logarithme d'un signal positif ergodique est lui-même ergodique, une propriété qui n'est évidemment pas générale pour toute application $\varphi$, et qui vient compléter l'arsenal des propriétés statistiques du logarithme $[4,5]$. 


\section{QUELQUES GENERALITES SUR L'ERGODICITE.}

2.1 - Soit un processus $Z_{t}$ stationnaire et du second ordre, $\mathrm{dF}_{1}(z)$ la loi de toute $Z_{t}$ et $d F_{2, \tau}\left(z_{1}, z_{2}\right)$ la loi conjointe de tout couple de variables $Z_{t}, Z_{t+\tau}$; par hypothèse ces lois admettent des moments du deuxième ordre, $\mathrm{dF}_{1}(\mathrm{z})$ ne dépend pas de $t$ et $\mathrm{dF}_{2, \tau}\left(\mathrm{z}_{1}, \mathrm{z}_{2}\right)$ ne dépend que de la durée $\tau$ qui sépare les variables $\mathrm{Z}_{4}$ et $Z_{t+\tau} ; \mathrm{dF}_{2, \tau}\left(\mathrm{z}_{1}, \mathrm{z}_{2}\right)$ est symétrique en $\mathrm{z}_{1}$ et $\mathrm{z}_{2}$, ses deux lois marginales sont $\mathrm{dF}_{1}\left(\mathrm{z}_{1}\right), \mathrm{dF}_{1}\left(\mathrm{z}_{2}\right)$. Soit $\mathrm{E}_{\mathrm{Z}}$ l'espérance aléatoire commune des $Z_{t}$ et $C(\tau)=\int\left(Z_{t}-E_{Z}\right)\left(Z_{t+\tau}-E_{Z}\right) d F_{2, \tau}$ la fonction de covariance centrée du processus.

Nous utilisons la définition suivante de l'ergodicité, la limite de la variable aléatoire $\frac{1}{\mathrm{~T}} \int_{0}^{T} \mathrm{Z}_{\mathrm{t}} \mathrm{dt}$ quand $\mathrm{T}$ tend vers plus l'infini est indépendante de la réalisation $\mathrm{Z}_{\mathfrak{\imath}}$ du processus, elle est égale à l'espérance $\mathrm{E}_{\mathbf{Z}}$ [Bass], la limite pouvant être au sens d'un des modes de convergence usuels en calcul des probabilités. Pour des processus stationnaires du second ordre nous avons la condition suffisante suivante : le processus est ergodique (pour l'espérance) en moyenne quadratique lorsque $\frac{1}{\mathrm{~T}} \int_{0}^{T} \mathrm{C}(\mathrm{t}) \mathrm{dt}$ tend vers zéro quand $T$ tend vers plus l'infini ; il suffit notamment que $C(t)$ ait une limite nulle quand $T$ tend vers plus l'infini pour qu'il en soit ainsi [6].

2.2 - Nous abordons l'ergodicité du signal par le biais des propriétés de la loi conjointe $\mathrm{dF}_{2, \tau}\left(\mathrm{z}_{1}, \mathrm{z}_{2}\right)$. Définition : la loi temporelle d'un signal vérifie $\mathrm{C} 1$ lorsque $\mathrm{dF}_{2, \tau}\left(\mathrm{z}_{1}, \mathrm{z}_{2}\right)$ converge simplement vers la loi produit des marges $\mathrm{dF}_{1}\left(\mathrm{z}_{1}\right) \mathrm{dF}_{1}\left(\mathrm{z}_{2}\right)$ quand $\tau$ tend vers plus l'infini, (en tout point de continuité).

Lemme 1: Si la loi du processus $Z_{\mathrm{t}}$ vérifie $\mathrm{C} 1$, le processus est ergodique.

En effet toutes les covariances centrées absolues $\int\left|Z_{t}-E_{Z}\right| Z_{\imath+\tau}-E_{Z} \mid d F_{2, \tau}$ sont définies, donc les fonctions positives $\left|Z_{\mathrm{t}}-\mathrm{E}_{\mathrm{Z}}\right|\left|\mathrm{Z}_{\imath+\tau}-\mathrm{E}_{\mathbf{Z}}\right| \mathrm{dF}_{2, \tau}$ intégrables.

Il existe une mesure $d M$ telle que $\left|Z_{t}-E_{Z}\right|\left|Z_{t+\tau}-E_{Z}\right| d F_{2, \tau}$ est majorée par $\left|Z_{t}-E_{Z}\right| Z_{t+\tau}-E_{Z} \mid d M$ ellemême intégrable, sinon (par l'absurde) $\mathrm{dF}_{2, \tau}$ n'a pas de marge du 2ème ordre.

D'après le théorème de Lebesgue il en résulte que l'intégrale de $\left(Z_{\imath}-E_{Z}\right)\left(Z_{\imath+\tau}-E_{Z}\right) d F_{2, \tau}\left(z_{1}, z_{2}\right)$ converge vers l'intégrale de $\left(Z_{1}-E_{Z}\right)\left(Z_{2}-E_{Z}\right) d F_{1}\left(z_{1}\right) d F_{1}\left(z_{2}\right)$, c'est à dire vers le produit de deux intégrales simples qui sont nulles par construction.

Cela signifie que la covariance centrée $C(\tau)$ tend vers zéro quand $\tau$ tend vers plus l'infini, et donc que le processus $\mathrm{Z}$ est ergodique pour l'espérance en moyenne quadratique.

Lemme 2 : Si la loi du processus $Z_{t}$ vérifie $C 1$ et si $\varphi$ est une application bornée, le processus $\varphi\left(Z_{\uparrow}\right)$ est stationnaire ergodique.

En effet le processus $\varphi\left(Z_{\downarrow}\right)$ est trivialement stationnaire, et puisque $\varphi$ est bornée l'espérance $E_{\varphi(Z)}=$ $\int \varphi(Z) d F_{1}(z)$ est définie, de mềme que les covariances centrées $\int\left(\varphi\left(Z_{\tau}\right)-E_{\varphi(Z)}\right)\left(\varphi\left(Z_{t+\tau}\right)-E_{\varphi(Z)}\right) d F_{2, \tau}$. Comme dans le Lemme 1 ces covariances convergent vers l'intégrale de $\left(\varphi\left(Z_{\nu}\right)-E_{\varphi(Z)}\right)\left(\varphi\left(Z_{t+\tau}\right)-E_{\varphi(Z)}\right)$ $\mathrm{dF}_{1}\left(\mathrm{z}_{1}\right) \mathrm{dF}_{1}\left(\mathrm{z}_{2}\right)$ quand $\tau$ tend vers plus l'infini, c'est à dire vers zéro.

Puisque la covariance centrée du processus $\varphi\left(Z_{t}\right)$ tend vers zéro quand t tend vers plus linfini, le processus est ergodique et vérifie $\frac{1}{T} \int_{0}^{T} \varphi\left(Z_{t}\right)$ dt tend vers $E_{\varphi(Z)}$ quand $T$ tend vers plus l'infini. 


\section{3 - LE CAS DU LOGARITHME.}

L'application logarithme n'étant pas bornée le Lemme 2 ne peut s'appliquer. Cependant la croissance du logarithme aux voisinages de l'origine et de plus l'infini étant faible, une hypothèse simple sur la loi $\mathrm{dF}_{2, \tau}$ entraîne que tous les processus $\log Z_{t}$ et $\left(\log Z_{V}\right)^{q}$ sont ergodiques.

\section{1 - La caractérisation d'une loi conjointe sur $\mathbf{R}^{+2}$.}

Soit $\mathrm{dF}_{X Y}(x, y)$ une loi conjointe de variables aléatoires positives $X$ et $Y$, et $\mathrm{I}_{X Y}\left(r_{1}, r_{2}\right)=E\left(X^{r 1} Y^{r^{2}}\right)=$ $\int \mathrm{x}^{\mathrm{r} 1} \mathrm{y}^{\mathrm{r} 2} \mathrm{dF}_{\mathrm{XY}}(\mathrm{x}, \mathrm{y})$, une application en fonction des paramètres $\mathrm{r}_{1}$ et $\mathrm{r}_{2}$ réels ; par construction elle est définie au-moins pour $r_{1}$ et $r_{2}$ nuls.

Soit la partition suivante de $\mathrm{R}^{+2}$ en 4 régions :

(1) : $X$ et $Y \geq 1$;

(2) : $X$ et $Y$ compris entre 0 et 1 ;

(3) : $X \geq 1, Y$ compris entre 0 et 1 ;

(4) : $Y \geq 1, X$ compris entre 0 et 1 ;

avec laquelle avec des notations évidentes $I_{X Y}\left(r_{1}, r_{2}\right)=I_{(1)}\left(r_{1}, r_{2}\right)+I_{(2)}\left(r_{1}, r_{2}\right)+I_{(3)}\left(r_{1}, r_{2}\right)+I_{(4)}\left(r_{1}, r_{2}\right)$.

Lemme 3: Si $\mathrm{I}_{X Y}\left(\mathrm{r}_{1}, \mathrm{r}_{2}\right)$ est définie pour $\mathrm{r}_{1}$ et $\mathrm{r}_{2}$ positifs, elle est définie pour $\mathrm{r}_{1}{ }^{\prime}$ et $\mathrm{r}_{2}{ }^{\prime}$ positifs respectivement inférieurs ou égaux à $r_{1}$ et $r_{2}$; si elle est définie pour $r_{1}$ et $r_{2}$ négatifs, elle l'est pour $r_{1}{ }^{\prime}$ et $r_{2}{ }^{\prime}$ négatifs respectivement supérieurs ou égaux à $r_{1}$ et $r_{2}$; si elle est définie pour $r_{1}$ positif et $r_{2}$ négatif, elle l'est pour $r_{1}{ }^{\prime}$ positif inférieur ou égal à $r_{1}$ et $r_{2}{ }^{\prime}$ négatif supérieur ou égal à $r_{2}$; si elle est définie pour $r_{1}$ négatif et $r_{2}$ positif, elle l'est pour $r_{1}{ }^{\prime}$ négatif supérieur ou égal à $r_{1}$ et $r_{2}{ }^{\prime}$ positif inférieur ou égal à $r_{2}$.

En effet

- dans le cas $n^{\circ} 1$ on a

$$
\begin{aligned}
& 0 \leq I_{X Y}\left(r_{1}{ }^{\prime}, r_{2}\right) \leq I_{(1)}\left(r_{1}, r_{2}\right)+I_{(2)}(0,0)+I_{(3)}\left(r_{1}, 0\right)+I_{(4)}\left(0, r_{2}\right), \\
& 0 \leq I_{X Y}\left(r_{1}{ }^{\prime}, r_{2}\right) \leq I_{(1)}(0,0)+I_{(2)}\left(r_{1}, r_{2}\right)+I_{(3)}\left(0, r_{2}\right)+I_{(4)}\left(r_{1}, 0\right), \\
& 0 \leq I_{X Y}\left(r_{1}{ }^{\prime}, r_{2}\right) \leq I_{(1)}\left(r_{1}, 0\right)+I_{(2)}\left(0, r_{2}\right)+I_{(3)}\left(r_{1}, r_{2}\right)+I_{(4)}(0,0), \\
& 0 \leq I_{X Y}\left(r_{1}{ }^{\prime}, r_{2}\right) \leq I_{(1)}\left(0, r_{2}\right)+I_{(2)}\left(r_{1}, 0\right)+I_{(3)}(0,0)+I_{(4)}\left(r_{1}, r_{2}\right) .
\end{aligned}
$$

Définition : on pose $r_{X M}$ et $r_{Y M}$ les bornes supérieures respectives des paramètres $r_{1}$ et $r_{2}$ positifs, et $r_{X m}$ et $r_{Y m}$ les bornes inférieures respectives de $r_{1}$ et $r_{2}$ négatifs tels que $I_{X Y}\left(r_{1}, r_{2}\right)$ est définie pour $r_{X m} \leq r_{1} \leq$ $r_{X M}$ et $r_{Y m} \leq r_{2} \leq r_{Y M}$; les bornes $r_{M}$ sont positives ou nulles, les bornes $r_{m}$ négatives ou nulles.

Lemme 4 : $I_{X Y}\left(r_{1}, r_{2}\right)=E\left(X^{r 1} Y^{r 2}\right)$ est défini sur un intervalle ]- $a_{1} a_{1}\left[\right.$ pour $\left.r_{1},\right]-a_{2} a_{2}\left[\right.$ pour $r_{2}, a_{1}$ et $a_{2}$ positifs, si et seulement si les produit $r_{X M} r_{X m}$ et $r_{Y M} r_{Y m}$ sont différents de zéro.

En effet il suffit de prendre $a_{1}=\inf \left(r_{X M},-r_{X m}\right)$ et $a_{2}=\inf \left(r_{Y M},-r_{Y m}\right)$, réciproquement $r_{X M} \geq a_{1}, r_{Y M} \geq$ $a_{2}, r_{X m} \leq-a_{1}$ et $r_{Y m} \leq-a_{2}$

Corollaire : Il en est de même pour $\mathrm{r}_{1}$ et $\mathrm{r}_{2}$ appartenant à un même intervalle ]-a $\mathrm{a}[$.

Définition : une distribution conjointe sur $R^{+2}$ vérifie la condition $C 2$ lorsque $I_{X Y}\left(r_{1}, r_{2}\right)$ est définie sur ]-a $a\left[^{2}\right.$ avec a $>0$. 


\section{2 - Les moments de $\log X$ et $\log Y$.}

Propriété 1 : Si la loi de $X, Y$ vérifie $C 2$ la loi conjointe des variables transformées $\log X$ et $\log Y$ admet des moments de tous ordres positifs.

En effet les moments d'ordre $k_{1}$ et $k_{2}$ positifs sont définis par $E\left((\log X)^{k 1}(\log Y)^{k 2}\right)=\int(\log x)^{k 1}$ $(\log y)^{k 2} \mathrm{dF}_{X Y}(\mathrm{x}, \mathrm{y})$, et soit la partition suivante $\left\{\left[0 \eta\left[\cup\left[\eta \mathrm{A}\left[\cup\left[\mathrm{A}+\infty[\}^{2}\right.\right.\right.\right.\right.\right.$ de $\mathrm{R}^{+2}$ avec $0<\eta<1<\mathrm{A}$;

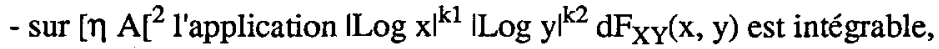
- sur chacune des 8 autres parties $(\log x)^{k 1}(\log y)^{k 2}$ garde un signe constant et n'est pas bornée, cependant dans chaque cas elle peut se factoriser sous la forme $x^{\delta 1}(\log x)^{k 1} y^{\delta 2}(\log x)^{k 2} x^{-\delta 1} y^{-\delta 2}$ avec $\delta_{1}$ et $\delta_{2}$ compris entre -a et $+\mathrm{a}$, et on peut prendre $\eta$ et A tels que l'application $x^{\delta 1}(\log x)^{k 1} y^{\delta 2}(\log x)^{k 2}$ peut être aussi petite que l'on veut et que $x^{-\delta 1} y^{-\delta 2} d F_{X Y}(x, y)$ est intégrable.

\section{3 - Retour aux processus.}

Le Lemme 1 et $\mathrm{C} 1$ concernent les processus, la Propriété 1 et $\mathrm{C} 2$ les lois conjointes dans $\mathrm{R}^{+2}$, en les associant il en résulte :

Propriété 2 : Si la loi $F_{2, \tau}$ d'un processus stationnaire positif $Z_{t}$ vérifie les conditions $\mathrm{C} 1$ et $C 2, Z_{t}$ est ergodique pour l'espérance en moyenne quadratique et il en est de même pour tout processus transformé $\left(\log Z_{\mathfrak{t}}\right)^{\mathrm{q}}$, q positif, on a ainsi limite $\frac{1}{\mathrm{~T}} \int_{0}^{\mathrm{T}}\left(\log Z_{\mathrm{u}}\right)^{\mathrm{q}} \mathrm{du}=\mathrm{E}\left\{\left(\log \mathrm{Z}_{\mathfrak{t}}\right)^{\mathrm{q}}\right\}$ quand $\mathrm{T}$ tend vers plus l'infini.

\section{APPLICATION.}

Nous obtenons ainsi un résultat technique sur le logarithme qui prolonge les propriétés précédentes $[4,5]$, et qui est intéressant en lui-même, en théorie du signal par exemple. A côté de cela il présente une grande importance en Acoustique de l'environnement sous réserve que les accroissements à l'origine des niveaux de bruit équivalents $\Delta\left\{\int_{\mathrm{p}_{\mathrm{u}}}{ }^{2} \mathrm{du}\right\}$ sur tous intervalles $[\mathrm{t} \mathrm{t}+\mathrm{h}]$ peuvent se mettre sous la forme d'un processus $Z_{\mathrm{t}}$ qui vérifie les deux conditions précédentes $\mathrm{C} 1$ et $\mathrm{C} 2$; auquel cas le résultat ci-contre, et donc l'ergodicité, s'étendent aux Log $\Delta\left\{\int \mathrm{p}_{\mathrm{u}}{ }^{2} \mathrm{du}\right\}$ et aux Leq $\mathrm{q}_{\mathrm{t}, \mathrm{i}}$ successifs sur les intervalles $\left[\mathrm{t}_{0}+(\mathrm{i}-1) \mathrm{h} \mathrm{t}_{0}+\mathrm{ih}\right]$.

C'est précisément ce qui se passe avec un grand nombre de PAS positifs (des travaux en cours à la suite de [3]). Par conséquent la Propriété 2 ci-dessus se révèle un point de passage utile pour l'ergodicité des $\mathrm{Leq}_{\Delta \mathrm{t}, \mathrm{i}}$ successifs dans le cadre de tels PAS, des processus qui sont plus riches et nombreux que les seuls PAIS, et qui élargissent l'ensemble des situations d'environnement routier où ils peuvent intervenir.

\section{Références :}

[1] Bouleau N., 1988, Processus stochastiques et applications, Hermann.

[2] Maurin M., 1991, A propos de la modélisation des niveaux de bruit, Revue de Statistique Appliquée, XXXIX $\mathrm{n}^{\circ} 2,69-74$.

[3] Maurin M., 1993a, Une classe de processus pour modéliser les niveaux de bruit de l'Environnement, Traitement du Signal, volume $10 \mathrm{n}^{\circ} 1,29-39$.

[4] Maurin M., 1993b, Quelques propriétés de la transformation logarithme en Probabilités, Congrès de l'ASU, Vannes.

[5] Maurin M., 1993c, Sur un nouvel intérêt du logarithme en acoustique statistique, à paraitre dans Acustica, $n^{\circ}$ 79.

[6] Bass J., 1974, Eléments de calcul des Probabilités, Masson. 